\title{
Bounds for Generalized Thrackles
}

\author{
G. Cairns and Y. Nikolayevsky \\ Department of Mathematics, La Trobe University, \\ Melbourne, Victoria 3083, Australia \\ \{G. Cairns, Y. Nikolayevsky\}@ latrobe.edu.au
}

\begin{abstract}
A thrackle (resp. generalized thrackle) is a drawing of a graph in which each pair of edges meets precisely once (resp. an odd number of times). For a graph with $n$ vertices and $m$ edges, we show that, for drawings in the plane, $m \leq \frac{3}{2}(n-1)$ for thrackles, while $m \leq 2 n-2$ for generalized thrackles. This improves theorems of Lovász, Pach, and Szegedy. The paper also examines thrackles in the more general setting of drawings on closed surfaces. The main result is: a bipartite graph $G$ can be drawn as a generalized thrackle on a closed orientable connected surface if and only if $G$ can be embedded in that surface.
\end{abstract}

\section{Introduction}

Let $G$ be a finite graph with $n$ vertices and $m$ edges, and suppose that $G$ is simple; that is, it has no loops or multiple edges. A thrackle of $G$ is a drawing $\mathcal{T}(G)$ of $G$ in the plane, where the edges are represented by Jordan arcs, such that each pair of edges meets precisely once, either at a vertex or at a proper crossing. (See [LPS] for definitions of drawing and proper crossing. Thrackles are mentioned in [CFG] and [PA].) Conway's celebrated thrackle conjecture is: $m \leq n$ (see [Wo1], [Wo2], [GR], [PRS], and [Ri]). A natural generalization of the notion of a thrackle is obtained by relaxing the condition that each pair of edges meets precisely once, and assuming only that each pair of edges meets an odd number of times. This gives rise to the notion of a generalized thrackle [Wo2]. Lovász et al. proved:

\section{Theorem 1 [LPS].}

(a) for thrackles, $m \leq 2 n-3$,

(b) for generalized thrackles, $m \leq 3 n-4$,

(c) a bipartite graph can be drawn as a generalized thrackle if and only if it is planar. 
We give the following improvement:

\section{Theorem 2.}

(a) for thrackles, $m \leq \frac{3}{2}(n-1)$,

(b) for generalized thrackles, $m \leq 2 n-2$.

We give examples below which show that the bound in Theorem 2(b) is sharp. In fact, our main focus in this paper is the study of generalized thrackles on surfaces of arbitrary genus, in the obvious sense. It was proved in [GR] that every finite graph can be thrackled on some surface. Our main result is:

Theorem 3. A bipartite graph $G$ can be drawn as a generalized thrackle on a closed orientable connected surface $M_{g}$ of genus $g$ if and only if $G$ can be embedded in $M_{g}$.

This has the corollary:

Corollary. For a bipartite generalized thrackle on $M_{g}$, one has $m \leq 2 n-4+4 g$.

This bound is sharp: for example, the minimal genus embedding of the complete bipartite graph $K_{2 p, 2 q}$ has $m=2 n-4+4 g$, by Ringel's theorem (see Theorem 4.5.3 of $[\mathrm{GT}])$.

The strategy employed in the proof of our results is to use $\mathbb{Z}_{2}$-intersection forms, and to reduce the problem to that of bipartite generalized thrackles. Thus the arguments are entirely about generalized thrackles. The additional improvement for thrackles is due solely to the fact that in the plane, thrackles have no 4-cycles [Wo1]. The main ideas in this paper are most easily described for generalized thrackles in the plane; here we show that Conway doubling on an odd cycle produces a bipartite graph $G^{\prime}$ (see Lemma 2). Furthermore, $G^{\prime}$ can be embedded in the plane so that the even cycle, resulting from the Conway doubling, bounds a face in the associated cellular decomposition (see Lemma 4).

The paper is organized as follows. In Section 1 we recall some facts about $\mathbb{Z}_{2}$-intersection forms and the Conway doubling procedure. In Section 2 we prove Theorem 3, and we give examples which show that Theorem 3 does not extend to arbitrary graphs. In Section 3 we obtain Theorem 2 and the corollary as special cases of Theorem 4, which is a slightly stronger result, and we give examples which show that the bound of Theorem 2(b) is sharp. The paper concludes in Section 4 with some remarks.

In what follows, $M_{g}$ denotes a closed oriented connected surface of genus $g$ and $G$ is a finite simple graph with $n$ vertices and $m$ edges.

\section{Intersection Homology and Conway Doubling}

First recall that the intersection form on $M_{g}$ is the unique nondegenerate bilinear map

$$
\Omega_{M_{g}}: H_{1}\left(M_{g}, \mathbb{Z}_{2}\right) \times H_{1}\left(M_{g}, \mathbb{Z}_{2}\right) \rightarrow \mathbb{Z}_{2}
$$


having the following property: if $\gamma_{1}$ and $\gamma_{2}$ are closed curves in $M_{g}$ which intersect in a finite number $k$ of transverse crossings, then $\Omega_{M_{g}}\left(\gamma_{1}, \gamma_{2}\right)=k \bmod 2$. Clearly, $\Omega_{M_{g}}$ is symmetric and $\Omega_{M_{g}}(\gamma, \gamma)=0$ for all closed curves $\gamma$. (See [ST] or [DFN] for further details. See $[\mathrm{Fu}]$ for an introductory account of intersection forms with values in $\mathbb{Z}$.)

Suppose that $\mathcal{T}: G \rightarrow M_{g}$ is a drawing of $G$, and that $c_{1}$ and $c_{2}$ are cycles in $G$. Recall that if $v$ is a vertex of $G$, then the rotation diagram at $\mathcal{T}(v)$ is the cyclic order of the edges of $G$ incident to $v$ determined by $\mathcal{T}$ and the orientation of $M_{g}$. The rotation system of $\mathcal{T}$ is the set of rotation diagrams of the vertices of $G$ (see [GT]). We want to relate $\Omega_{M_{g}}\left(\mathcal{T}\left(c_{1}\right), \mathcal{T}\left(c_{2}\right)\right)$ to the rotation system of $\mathcal{T}$. The following notion depends only on the rotation system:

Definition 1. The crossing number $\sigma_{\mathcal{T}}\left(c_{1}, c_{2}\right)$ is defined as follows: choose an orientation for $c_{1}$ and $c_{2}$, and consider the set $S$ of vertices in the boundary of $c_{1} \cap c_{2}$. Let $v \in S$. If $v$ is isolated in $c_{1} \cap c_{2}$, set

$$
\sigma_{\mathcal{T}}(v)= \begin{cases}1 & \text { if } c_{1} \text { and } c_{2} \text { cross transversally at } v \\ 0 & \text { otherwise }\end{cases}
$$

If $v$ is not isolated in $c_{1} \cap c_{2}$, set

$$
\sigma_{\mathcal{T}}(v)=\left\{\begin{aligned}
\frac{1}{2} & \text { if } c_{2} \text { is positively oriented at } v \text { with respect to } c_{1}, \\
-\frac{1}{2} & \text { if } c_{2} \text { is negatively oriented at } v \text { with respect to } c_{1},
\end{aligned}\right.
$$

where the sign convention is shown in Fig. 1. Then $\sigma_{\mathcal{T}}\left(c_{1}, c_{2}\right)=\sum_{v \in S} \sigma_{\mathcal{T}}(v) \bmod (2)$.

Let $l$ denote the $\mathbb{Z}_{2}$-length function on the 1 -chain complex of $G$; that is, given a path $c$ in $G, l(c)$ is the number $\bmod (2)$ of edges in $c$. The following result may be regarded as a generalization of Lemmas 2.2 and 2.3 of [LPS].

Lemma 1. Suppose that $\mathcal{T}: G \rightarrow M_{g}$ is a generalized thrackle, and that $c_{1}$ and $c_{2}$ are cycles in $G$. Then $\Omega_{M_{g}}\left(\mathcal{T}\left(c_{1}\right), \mathcal{T}\left(c_{2}\right)\right)=\sigma_{\mathcal{T}}\left(c_{1}, c_{2}\right)+l\left(c_{1}\right) \cdot l\left(c_{2}\right)+l\left(c_{1} \cap c_{2}\right)(\bmod 2)$.

Proof. Divide the edges of $c_{1}$ into four disjoint subsets: (a) $k_{1}$ edges contained in $c_{1} \cap c_{2}$, (b) $k_{2}$ edges which are not incident with $c_{1} \cap c_{2}$, (c) $k_{3}$ edges not contained in $c_{1} \cap c_{2}$
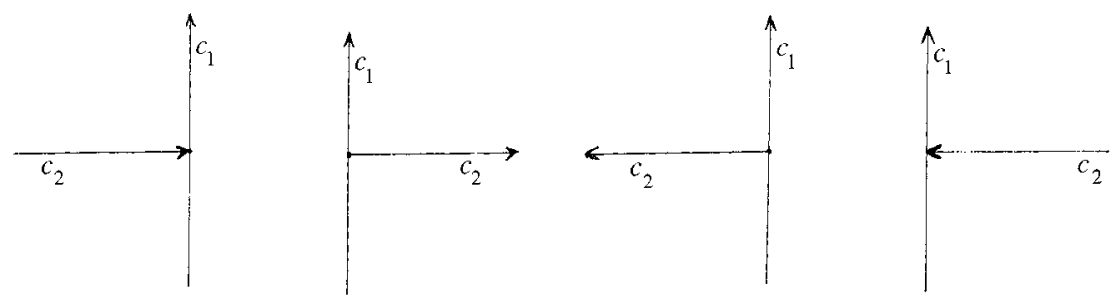

Fig. 1. Two $+\frac{1}{2}$ crossings and two $-\frac{1}{2}$ crossings. 

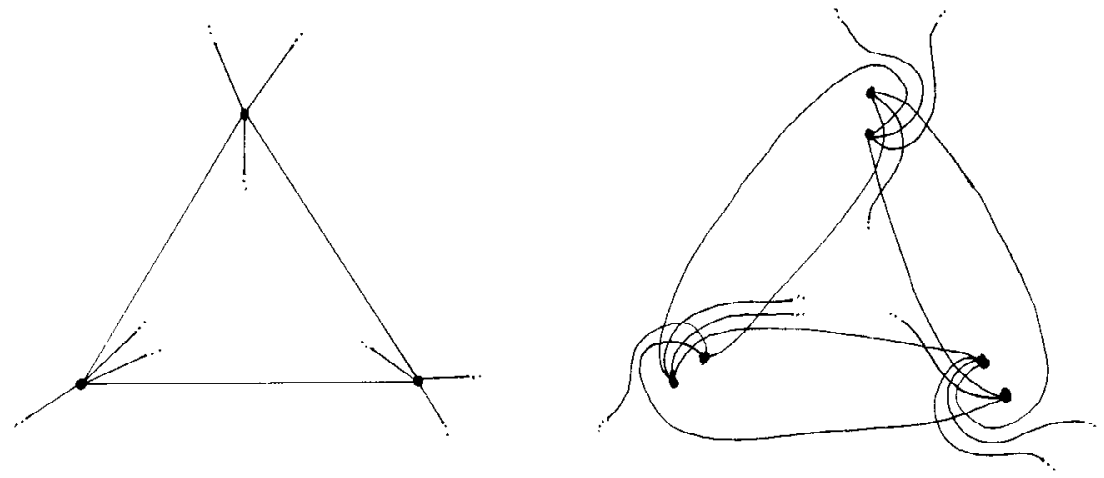

Fig. 2. Conway doubling on a 3-cycle: before and after.

which meet $c_{1} \cap c_{2}$ at exactly one vertex, and (d) $k_{4}$ edges not contained in $c_{1} \cap c_{2}$ which meet $c_{1} \cap c_{2}$ at exactly two vertices. Modulo 2, one has

$$
\begin{aligned}
\Omega_{M_{g}}\left(\mathcal{T}\left(c_{1}\right), \mathcal{T}\left(c_{2}\right)\right)= & \sigma_{\mathcal{T}}\left(c_{1}, c_{2}\right)+k_{1} \cdot\left(l\left(c_{2}\right)-3\right)+k_{2} \cdot l\left(c_{2}\right) \\
& +k_{3}\left(l\left(c_{2}\right)-2\right)+k_{4}\left(l\left(c_{2}\right)-4\right) \\
= & \sigma_{\mathcal{T}}\left(c_{1}, c_{2}\right)+k_{1}+\left(k_{1}+k_{2}+k_{3}+k_{4}\right) \cdot l\left(c_{2}\right) \\
= & \sigma_{\mathcal{T}}\left(c_{1}, c_{2}\right)+l\left(c_{1} \cap c_{2}\right)+l\left(c_{1}\right) \cdot l\left(c_{2}\right),
\end{aligned}
$$

as required.

Conway's doubling procedure allows one to duplicate a thrackled cycle [Wo1]. If the original cycle is odd, one ends up with a thrackled even cycle which is twice as long, and if it is even, one obtains a pair of disjoint even cycles of the same length which still form a (disconnected) thrackle. This procedure can be carried out not only for a separate cycle, but also for a cycle within a thrackled graph, or within a generalized thrackle, and the procedure can be made on any surface (see Fig. 2).

Let $\mathcal{T}: G \rightarrow M_{g}$ be a generalized thrackle.

Lemma 2. Suppose that $c_{1}$ is an odd cycle in $G$ such that $\Omega_{M_{g}}\left(\mathcal{T}\left(c_{1}\right), \mathcal{T}\left(c_{2}\right)\right)=0$ for all cycles $c_{2}$. Then Conway doubling on $c_{1}$ produces a bipartite graph.

Proof. Let $c_{1}$ be as in the statement of the lemma. Perform the Conway doubling procedure on $c_{1}$ : let $G^{\prime}$ be the resulting graph and let $c_{1}^{\prime}$ be the even cycle obtained from $c_{1}$. We claim that $G^{\prime}$ is bipartite. Suppose that $G^{\prime}$ has a cycle $c_{2}^{\prime}$. By reversing the Conway doubling procedure, one sees that $c_{2}^{\prime}$ comes from a cycle $c_{2}$ say, in $G$. Obviously,

$$
l\left(c_{2}^{\prime}\right)-l\left(c_{1}^{\prime} \cap c_{2}^{\prime}\right)=l\left(c_{2}\right)-l\left(c_{1} \cap c_{2}\right) .
$$

By Lemma 1, we have, modulo 2,

$$
\sigma_{\mathcal{T}}\left(c_{1}, c_{2}\right)=l\left(c_{1}\right) l\left(c_{2}\right)+l\left(c_{1} \cap c_{2}\right)=l\left(c_{2}\right)+l\left(c_{1} \cap c_{2}\right)
$$


and

$$
\sigma_{\mathcal{T}}\left(c_{1}^{\prime}, c_{2}^{\prime}\right)=l\left(c_{1}^{\prime}\right) l\left(c_{2}^{\prime}\right)+l\left(c_{1}^{\prime} \cap c_{2}^{\prime}\right)=l\left(c_{1}^{\prime} \cap c_{2}^{\prime}\right) .
$$

Notice that $\sigma_{\mathcal{T}}\left(c_{1}, c_{2}\right)=\sigma_{\mathcal{T}}\left(c_{1}^{\prime}, c_{2}^{\prime}\right)$ and so

$$
l\left(c_{2}\right)+l\left(c_{1} \cap c_{2}\right)=l\left(c_{1}^{\prime} \cap c_{2}^{\prime}\right) .
$$

So (1) and (2) give $l\left(c_{2}^{\prime}\right)=0(\bmod 2)$, as required.

We remark that the hypothesis in Lemma 2 is weaker than the assumption that $\mathcal{T}\left(c_{1}\right)$ is zero in $\mathbb{Z}_{2}$-homology. In particular, it holds in the plane. One of this paper's referees has informed us that in previous personal communication, Péter Hajnal had independently obtained Lemma 2 in the planar case, thus improving Theorem 1 (a) to $m \leq 1.75 n$.

\section{Proof of Theorem 3}

Let $G$ be a bipartite graph and let $V(G)=V_{1} \cup V_{2}$ be a splitting of the set of vertices of $G$ such that all the edges join elements of $V_{1}$ with elements of $V_{2}$.

First suppose that there is an embedding $f: G \rightarrow M_{g}$. For convenience, we use $f$ to identify $G$ with $f(G)$, so that we may regard $G$ as a subset of $M_{g}$. The following argument is similar to the one used in Theorem 1.4 of [LPS]. Choose a point $x$ in the complement of $G$, and for each point $y \in V_{1}$, join $x$ to $y$ by a simple arc such that the set of arcs thus obtained is mutually disjoint outside of $x$. By deforming these arcs if necessary, we may assume that they avoid $V_{2}$, and cross the edges of $G$ in proper crossings. Take a small closed $\varepsilon$-neighbourhood $D$ of the union of these $\operatorname{arcs}$ (see Fig. 3). So $D$ is homeomorphic to a disc, and the boundary of $D$ is a simple closed curve $\gamma$ which intersects every edge of $G$ an odd number of times. Let $\gamma^{\prime}$ be a curve on $M_{g} \backslash D$ which is sufficiently close to $\gamma$ that there are no vertices of $G$ between $\gamma$ and $\gamma^{\prime}$. Cut out the disc $D$, flip it over, and attach it back to the surface joining the edges in the annulus between $\gamma$ and $\gamma^{\prime}$ as shown on the Fig. 4. Notice that in the new drawing, any two non-incident edges $e_{i}(i=1,2)$ intersecting $\gamma q_{i}$ times respectively, intersect one another $q_{1} q_{2} \equiv 1(\bmod 2)$ times, while every pair of incident edges meet an even number of times. Now, taking small circles around each of the vertices of $G$ and performing the above procedure in each of the discs they bound, we obtain a new drawing in which

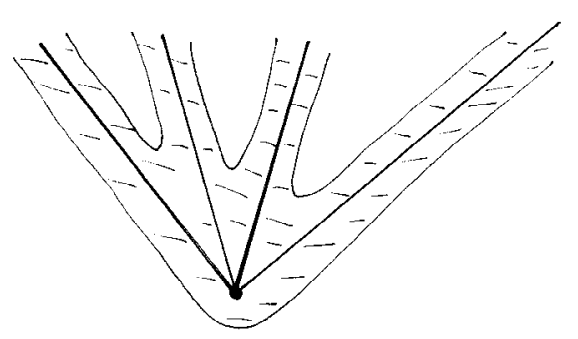

Fig. 3. The $\varepsilon$-neighbourhood $D$ in the vicinity of $x$. 

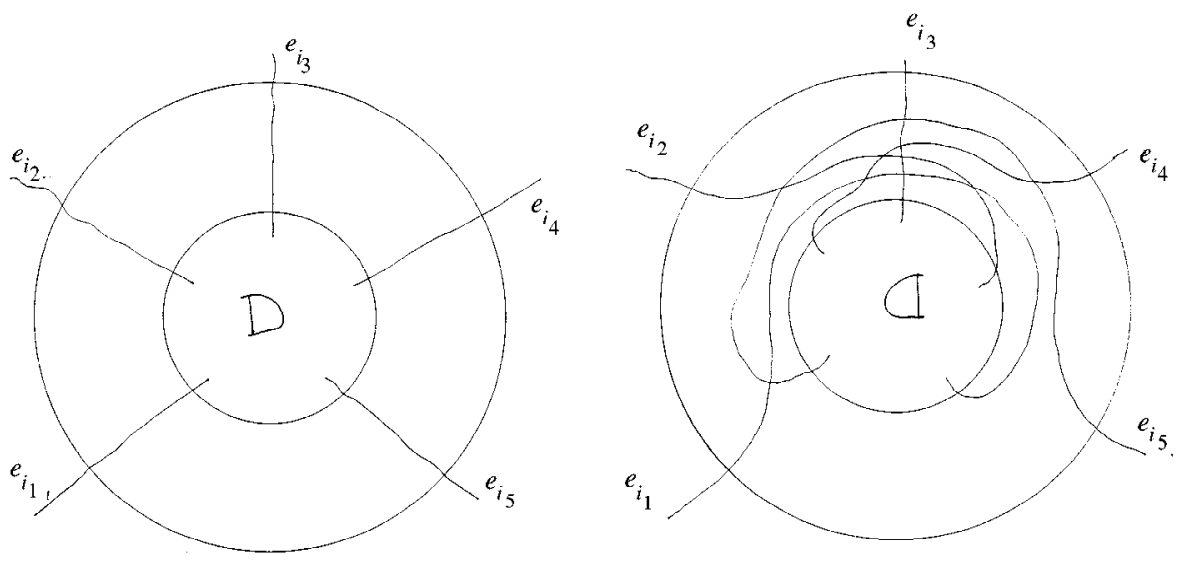

Fig. 4. Reattaching $D$ : before and after.

every pair of edges meets an odd number of times. It remains to modify the drawing so that each edge becomes free of self-intersections. This can be achieved edge by edge: for each edge, it suffices to choose a Wiener switching at each of the self-intersections such that the resulting crossing-free curve is connected (see Fig. 5). This is easily done by induction. The resulting drawing is a generalized thrackle.

Conversely, suppose that we have a generalized thrackle $\mathcal{T}: G \rightarrow M_{g}$. Proceed as in the first part of this proof: choose a point $x$ in the complement of $G$, and join $x$ to $V_{1}$ by a set of arcs. Perform the procedure shown Fig. 4 in a small $\varepsilon$-neighbourhood of these arcs, and then choose small discs around each of vertices of $G$ and repeat the procedure on each of these discs. One thus obtains a new drawing $\mathcal{D}: G \rightarrow M_{g}$, which is a $\mathbb{Z}_{2}$-embedding, in the following sense.

Definition 2. A $\mathbb{Z}_{2}$-embedding of a graph in $M_{g}$ is a drawing of the graph such that every pair of edges meets an even number of times, outside the vertex set.

It remains to prove the following lemma (notice that we are not assuming here that $G$ is bipartite).
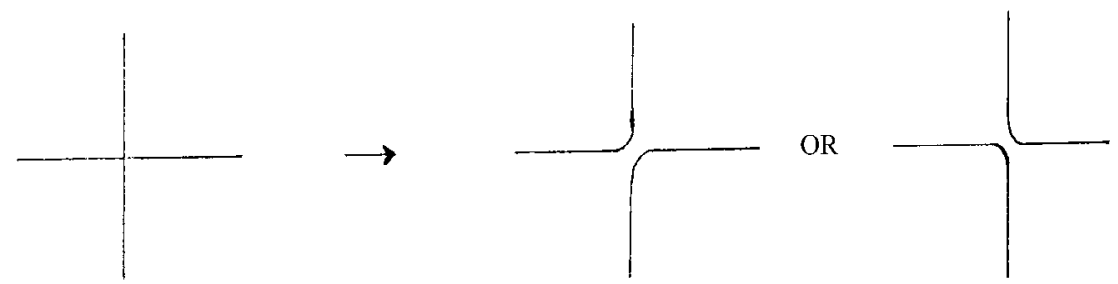

Fig. 5. Wiener switching: before and after. 

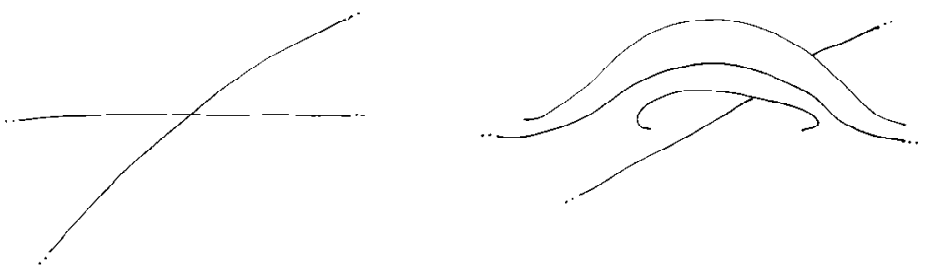

Fig. 6. Eliminating a crossing: before and after.

Lemma 3. A graph $G$ that can be $\mathbb{Z}_{2}$-embedded in $M_{g}$, can be embedded in $M_{g}$ with the same rotation system.

Proof of Lemma 3. Suppose that we have a $\mathbb{Z}_{2}$-embedding $\mathcal{D}: G \rightarrow M_{g}$. Obviously, as $\mathcal{D}$ is not a generalized thrackle, Lemma 1 does not apply. Instead one has

$$
\Omega_{M_{g}}\left(\mathcal{D}\left(c_{1}\right), \mathcal{D}\left(c_{2}\right)\right)=\sigma_{\mathcal{D}}\left(c_{1}, c_{2}\right) \quad(\bmod 2)
$$

for all cycles $c_{1}$ and $c_{2}$ in $G$. Now remove all the crossings in $\mathcal{D}(G)$ by attaching a handle at each crossing point (see Fig. 6), and let $S$ be the resulting closed surface. So we have an embedding $\mathcal{I}(G)$ of $G$ in $S$, but in general $S$ has higher genus than $M_{g}$. Notice that since the surgery has been conducted in the complement of some neighbourhood of the vertex set, $\mathcal{I}(G)$ and $\mathcal{D}(G)$ have the same rotation systems; in particular, $\sigma_{\mathcal{I}}=\sigma_{\mathcal{D}}$. Take a closed $\varepsilon$-neighbourhood $U$ of $\mathcal{I}(G)$; so $U \subset S$ is a compact surface, with boundary, containing $\mathcal{I}(G)$. Since $\mathcal{I}(G)$ is a deformation retract of $U$, we have $H_{1}\left(U, \mathbb{Z}_{2}\right)=H_{1}\left(\mathcal{I}(G), \mathbb{Z}_{2}\right)$ and $\Omega_{U}=\sigma_{\mathcal{I}}$. Attach discs to all the boundary components of $U$ and let $M^{\prime}$ be the resulting surface. If $G$ is not connected, then $M^{\prime}$ will not be connected; in this case, replace $M^{\prime}$ by the connected sum of its connected components. We now have an embedding $\mathcal{J}(G)$ of $G$ in a connected closed oriented surface $M^{\prime}$, and it remains to show that the genus $g^{\prime}$ of $M^{\prime}$ is not greater than that of $M_{g}$. Notice that by construction, the map $H_{1}\left(U, \mathbb{Z}_{2}\right) \rightarrow H_{1}\left(M^{\prime}, \mathbb{Z}_{2}\right)$ is surjective. Hence

$$
\begin{aligned}
g^{\prime}=\frac{1}{2} \operatorname{rank} H_{1}\left(M^{\prime}, \mathbb{Z}_{2}\right) & \leq \operatorname{rank} \Omega_{U} \\
& =\operatorname{rank} \sigma_{\mathcal{I}} \\
& =\operatorname{rank} \sigma_{\mathcal{D}} \\
& \leq \operatorname{rank} \Omega_{M_{g}} \quad \text { by (3) } \\
& =g,
\end{aligned}
$$

as required.

Examples. Figure 7 gives an example of a non-planar graph, homeomorphic to $K_{5}$, which can be drawn as a generalized thrackle in the plane. 

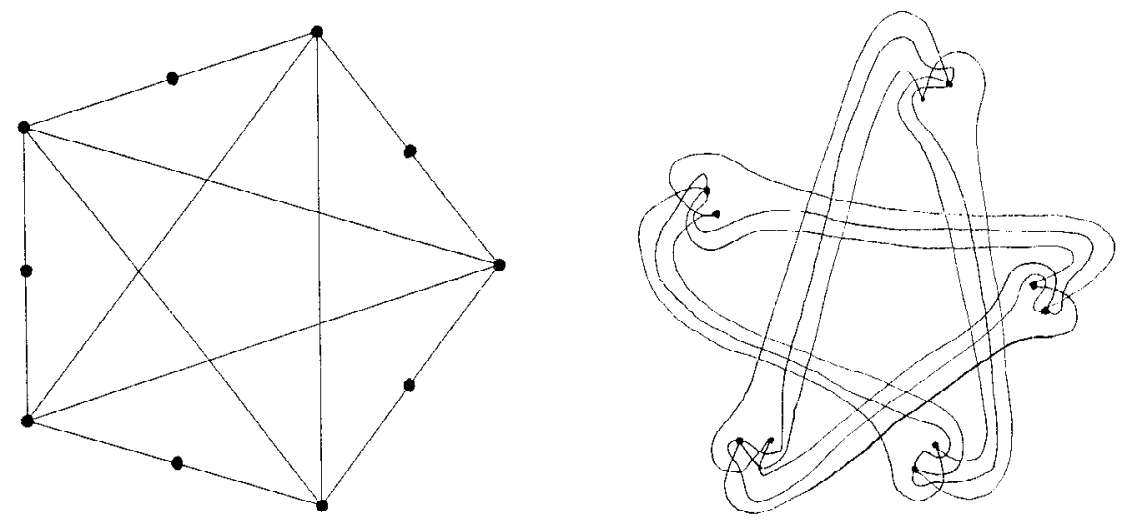

Fig. 7. A generalized thrackle homeomorphic to $K_{5}$.

On the other hand, there are planar graphs with $m \leq 2 n-2$ which cannot be drawn as generalized thrackles in the plane. For example, the wheel with four spokes, shown in Fig. 8, cannot be drawn as a generalized thrackle in the plane. Indeed, if it could be, then, by [LPS] or Lemma 1, the two 3-cycles to the left and right of the graph would necessarily cross each other transversally in a small neighbourhood of the vertex in the centre of the graph. However, the same reasoning applies to the two 3-cycles at the top and bottom of the graph. This leads to a contradiction. Similarly, the wheel with $2 k$ spokes cannot be drawn as a generalized thrackle in the plane. Curiously, wheels with an odd number of spokes can be drawn as generalized thrackles in the plane (see Fig. 12 below).

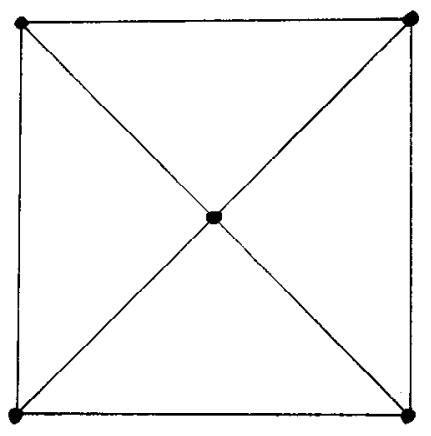

Fig. 8. Not a generalized thrackle in the plane. 


\section{Proof of Theorem 2 and the Corollary}

Let $k$ denote the number of connected components of $G$.

Theorem 4. Suppose that $\mathcal{T}: G \rightarrow M_{g}$ is a generalized thrackle.

(a) If $G$ is bipartite, then $m \leq 2 n-4 k+4 g$.

(b) If $G$ has an odd cycle $c_{1}$ such that $\Omega_{M_{g}}\left(\mathcal{T}\left(c_{1}\right), \mathcal{T}\left(c_{2}\right)\right)=0$ for all cycles $c_{2}$, then $m \leq 2 n-2 k+4 g$.

(c) If $G$ is a thrackle in the plane, then $m \leq \frac{3}{2}(n-k)$.

Proof. First suppose that $G$ is bipartite. By Theorem 3, $G$ can be embedded in $M_{g}$. So if $G$ has connected components $G_{1}, \ldots, G_{k}$, then, for each $i$, we have a cellular embedding of $G_{i}$ in a surface $S_{i}$ of genus $g_{i}$, with $g_{1}+\cdots+g_{k} \leq g$. Thus it suffices to treat the case where $G$ is connected and cellularly embedded in $M_{g}$. In this case, part (a) is a direct consequence of Euler's formula, as employed in [LPS]. Indeed, $2-2 g=f-m+n$, where $f$ is the number of faces in the cellular decomposition of $M_{g}$ determined by the embedding of $G$. As $G$ has no 2-cycles or 3-cycles, one has $2 m \geq 4 f$. Hence

$$
n=2-2 g-f+m \geq 2-2 g-m / 2+m=2-2 g+m / 2 .
$$

That is, $m \leq 2 n-4+4 g$. This proves part (a).

Now suppose that $G$ has an odd cycle $c_{1}$ such that $\Omega_{M_{g}}\left(\mathcal{T}\left(c_{1}\right), \mathcal{T}\left(c_{2}\right)\right)=0$ for all cycles $c_{2}$. Perform Conway doubling on $c_{1}$ : let $G^{\prime}$ be the resulting bipartite graph and let $c_{1}^{\prime}$ be the even cycle obtained from $c_{1}$. Let $\mathcal{J}: G^{\prime} \rightarrow M_{g}$ be the embedding given by the construction of Theorem 3. Once again, it suffices to treat the case where $G$ is connected and $G^{\prime}$ is cellularly embedded in $M_{g}$.

Lemma 4. $\mathcal{J}\left(c_{1}^{\prime}\right)$ bounds a face in the cellular decomposition of $M_{g}$ determined by the embedding of $G^{\prime}$.

Proof of Lemma 4. Since $M_{g}$ is oriented, it makes sense to talk of the "left" and "right" sides of a closed curve, at least locally. It suffices to show that the image in $M_{g}$ of the edges of $G^{\prime} \backslash c_{1}^{\prime}$ which are incident with $c_{1}^{\prime}$ all lie on the same side of $\mathcal{J}\left(c_{1}^{\prime}\right)$. Let $\mathcal{C}: G^{\prime} \rightarrow M_{g}$ be the generalized thrackle drawing of $G^{\prime}$ obtained from $\mathcal{T}(G)$ by Conway doubling on $c_{1}$. Notice that the edges of $G^{\prime} \backslash c_{1}^{\prime}$ which are incident with $c_{1}^{\prime}$ at some given vertex $v$, all lie on the same side of $\mathcal{C}\left(c_{1}^{\prime}\right)$, either to the left or to the right (see Fig. 2). Moreover, the position of the incident edges alternates, left-right-left, etc., as one moves from vertex to vertex along $\mathcal{C}\left(c_{1}^{\prime}\right)$ (see Fig. 2). By Lemma 2, $G^{\prime}$ is bipartite: let $V(G)=V_{1} \cup V_{2}$ be a splitting of the set of vertices of $G$ such that all the edges join elements of $V_{1}$ with elements of $V_{2}$. Now notice that when one constructs the embedding $\mathcal{J}\left(G^{\prime}\right)$, using the method employed in the proof of Theorem 3, one effectively reverses the orientation in some neighbourhood of $V_{2}$, while maintaining the orientation in some neighbourhood of $V_{1}$. Consequently, as one travels along $\mathcal{J}\left(c_{1}^{\prime}\right)$, the edges incident with $\mathcal{J}\left(c_{1}^{\prime}\right)$ all lie on the same side of $\mathcal{J}\left(c_{1}^{\prime}\right)$. 
It remains to see how the above lemma gives the required result. Let $f, m^{\prime}, n^{\prime}$ be respectively the number of faces, edges and vertices in the cellular decomposition of $M_{g}$ determined by the embedding of $G^{\prime}$. Suppose that $c_{1}$ is a $p$-cycle. So $c_{1}^{\prime}$ is a $2 p$ cycle, $n^{\prime}=n+p$ and $m^{\prime}=m+p$. As $G$ has no 2-cycles or 3-cycles, one has $2 m^{\prime} \geq 4(f-1)+2 p$. Hence

$$
n^{\prime}=2-2 g-f+m^{\prime} \geq 1-2 g+\frac{m^{\prime}+p}{2} .
$$

That is, $m=m^{\prime}-p \leq 2 n^{\prime}-2 p-2+4 g=2 n-2+4 g$. This proves part (b). To prove part (c), just repeat the calculation using the additional fact that $G$ has no 4-cycles. This completes the proof of Theorem 4.

Notice that Theorem 2 follows immediately from Theorem 4 , since in the plane every cycle is $\mathbb{Z}_{2}$-null homologous. The corollary follows immediately from Theorem 4(a).

Examples. We first describe a useful construction. Let $\mathcal{T}: G \rightarrow M_{g}$ be a generalized thrackle. We say that two edges $e_{1}$ and $e_{2}$ of $G$ are neighbouring if they share a common vertex $v$ and if $e_{1}$ and $e_{2}$ are consecutive in the cyclic order of edges of $\mathcal{T}(G)$ at $\mathcal{T}(v)$. Figure 9 shows how one can add a 2-path joining the endpoints of neighbouring edges so that the resulting drawing is still a generalized thrackle. Notice that Fig. 7 is obtained by adding five 2-paths to the standard pentagonal musquash [Wo1].

Figure 10 gives a drawing of $K_{4}$ as a generalized thrackle in the plane. Figure 11 shows that by adding 2-paths, one can construct a generalized thrackle in the plane having $n$ vertices and $2 n-4$ edges, for any number $n \geq 4$. Another example showing that the bound in Theorem 2(b) is sharp is given by the wheel with $(2 k+1)$ spokes. Figure 12 shows the wheel with five spokes and its representation as a generalized thrackle; this diagram is to be understood as follows: five edges meet at a vertex at infinity, and each pair of these edges cross precisely once in a small neighbourhood of infinity. This example is built on the standard pentagonal musquash [Wo1]. This same construction can be effected using the standard $(2 k+1)$-gonal musquash, for any $k \geq 1$. Notice that Figure 10 is the case $k=1$.
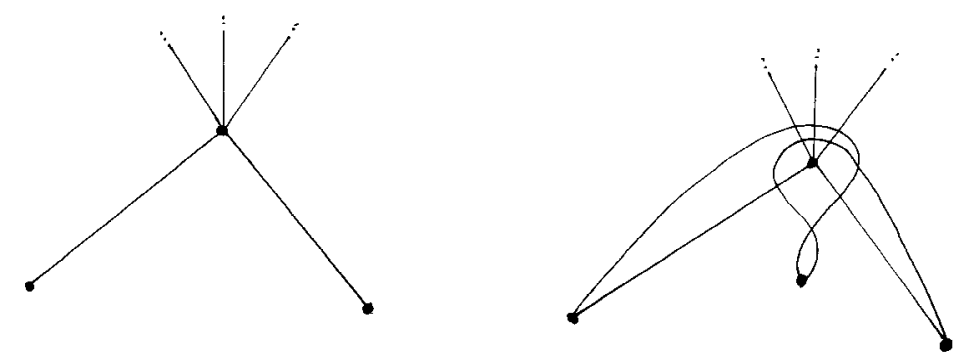

Fig. 9. Attaching a 2-path to a pair of neighbouring edges. 


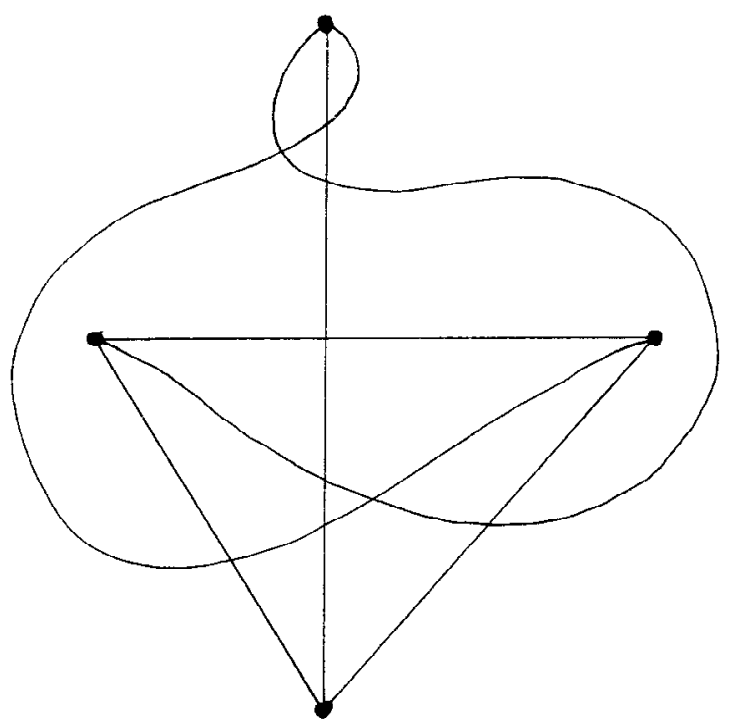

Fig. 10. Generalized thrackle of $K_{4}$ in the plane.

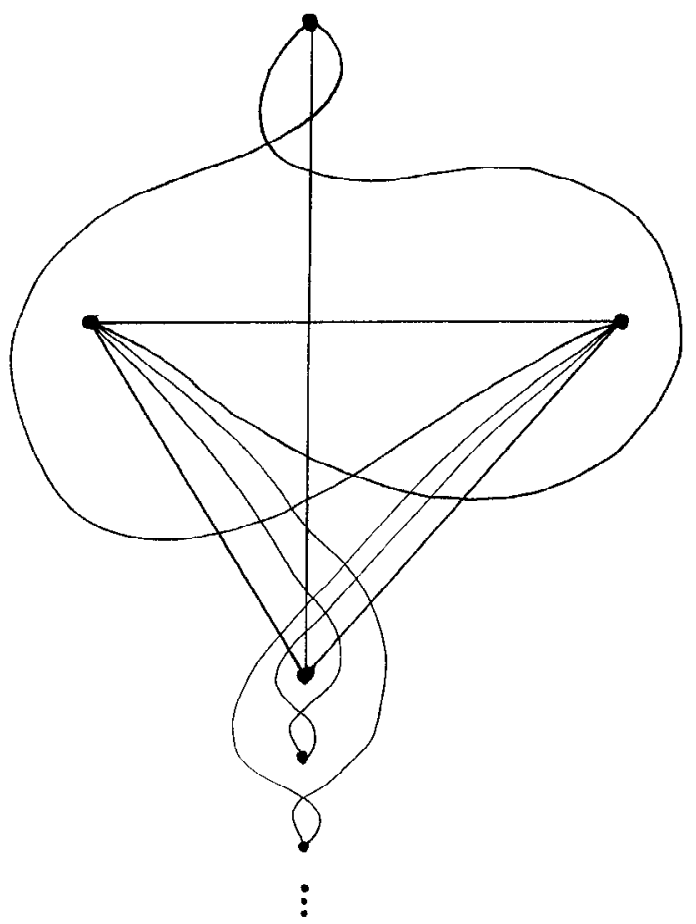

Fig. 11. Planar generalized thrackle with $m=2 n-2$. 

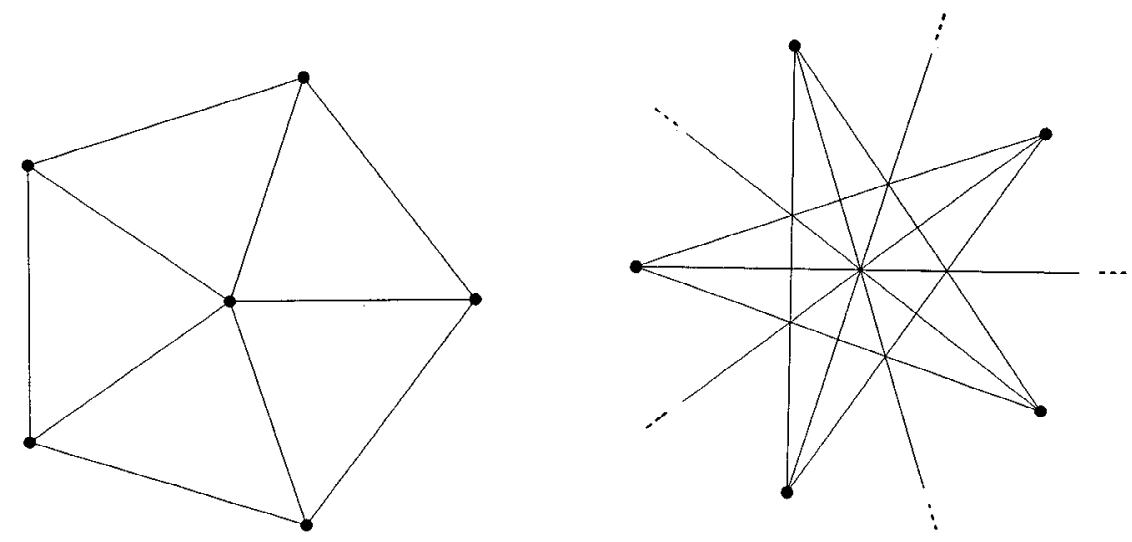

Fig. 12. Another planar generalized thrackle with $m=2 n-2$.

\section{Remarks}

As we have seen above, Theorem 4 provides sharp bounds for generalized thrackles on $M_{g}$ where $G$ has no odd cycles, and where there is an odd cycle which is zero in $\mathbb{Z}_{2}$-homology. For arbitrary generalized thrackles, one has $m \leq 4 n-8+8 g$, since every graph can be made bipartite by removing no more than half of its edges. However, this bound seems unduly coarse. We have found no counterexample to the following:

Conjecture 1. If $\mathcal{T}: G \rightarrow M_{g}$ is a generalized thrackle, then $m \leq 2 n-2+4 g$.

In fact, it does not seem unreasonable to hope to obtain a complete classification of those graphs which can be drawn as generalized thrackles in the plane.

Our final remarks concern thrackles, as opposed to generalized thrackles. In [CFG], the authors remark: "We may consider analogous constructions on other surfaces, and presumably expect (with obvious notation) the appropriate conjecture to be that $\max (m-$ $n$ ) depends on the genus of the surface." The following conjecture seems to be the obvious one, although as far as we are aware, it has not previously appeared explicitly in the literature:

Conjecture 2. If $\mathcal{T}: G \rightarrow M_{g}$ is a thrackle, then $m \leq n+2 g$.

Observe that, for any given genus $g$, there exists an example for which the bound $m=n+2 g$ is attained. This can be done inductively using a procedure similar to that employed in Fig. 14 of [Wo1]; one chooses an example for which the bound is attained on a surface of genus $g-1$, and then adds a handle to the surface and replaces an edge by the system of five edges shown in Fig. 13. This increases the number of vertices by two and the number of edges by four.

Conjecture 2 can be verified for graphs with very few vertices. First, recall that thrackles in the plane have no 4-cycles [Wo1]. Moreover, they have at most one 3-cycle; 

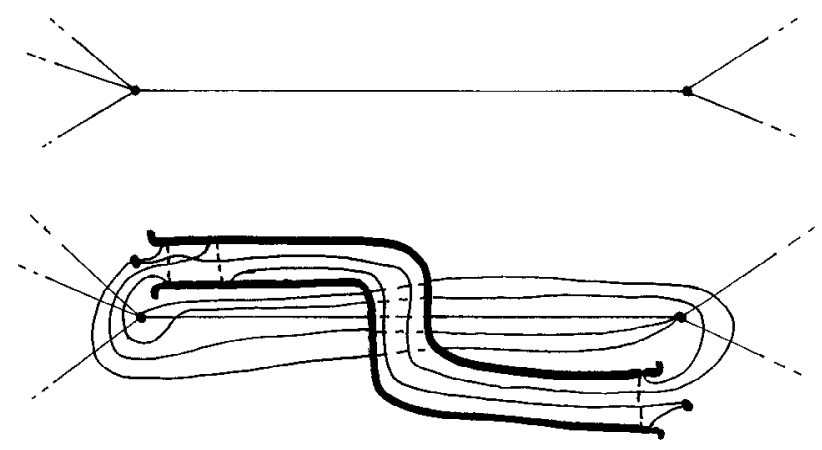

Fig. 13. Before and after.

indeed, if a thrackle in the plane had two 3-cycles, $c_{1}$ and $c_{2}$ say, then $c_{1}$ and $c_{2}$ must have nontrivial intersection (by [LPS]), but they cannot share a common edge (since otherwise there would be a 4-cycle) and it is easy to see that the case of a single common vertex is also impossible. In higher genus, one has:

Lemma 5. Suppose that $\mathcal{T}: G \rightarrow M_{g}$ is a thrackle.

(a) If $c \subset G$ is a 4-cycle, then $\mathcal{T}(c)$ is nontrivial in $\mathbb{Z}_{2}$-homology.

(b) If $c_{1}, c_{2} \subset G$ are 3 -cycles, then $\mathcal{T}\left(c_{1}\right)$ and $\mathcal{T}\left(c_{2}\right)$ are not $\mathbb{Z}_{2}$-homologous.

Proof. (a) Let $c=\{1234\}$ be a thrackled 4-cycle on $M_{g}$. Let $a=12 \cap 34, b=23 \cap 14$. Then the triangles $12 b$ and $34 b$ have exactly one point of the transversal crossing (namely, the point $a$ ). They cannot represent the same class in $H_{1}\left(M_{g}, \mathbb{Z}_{2}\right)$ and therefore their sum is nontrivial.

(b) Suppose that $G$ consists of two 3-cycles $c_{1}$ and $c_{2}$, and that $\mathcal{T}\left(c_{1}\right)$ and $\mathcal{T}\left(c_{2}\right)$ are $\mathbb{Z}_{2}$-homologous. Since $G$ is simple, $c_{1}$ and $c_{2}$ are either disjoint, share a single common edge, or share a single common vertex. First notice that if $c_{1}$ and $c_{2}$ shared a single common edge, then their sum would be a 4-cycle whose image in $M_{g}$ would be trivial in $\mathbb{Z}_{2}$-homology. This would contradict part (a). So $c_{1}$ and $c_{2}$ are either disjoint, or share a single common vertex. As $\mathcal{T}\left(c_{1}\right)$ and $\mathcal{T}\left(c_{2}\right)$ are $\mathbb{Z}_{2}$-homologous, $\mathcal{T}\left(c_{1}\right)$ and $\mathcal{T}\left(c_{2}\right)$ must have zero intersection number. So Lemma 1 gives

$$
0=\Omega_{M_{g}}\left(\mathcal{T}\left(c_{1}\right), \mathcal{T}\left(c_{2}\right)\right)=\sigma_{\mathcal{T}}\left(c_{1}, c_{2}\right)+1+l\left(c_{1} \cap c_{2}\right) .
$$

If $c_{1}$ and $c_{2}$ were disjoint, then one would have $\sigma_{\mathcal{T}}\left(c_{1}, c_{2}\right)=0$ and $l\left(c_{1} \cap c_{2}\right)=0$, which contradicts (4). So $c_{1}$ and $c_{2}$ share a single common vertex. Hence (4) gives $\sigma_{\mathcal{T}}\left(c_{1}, c_{2}\right)=1$. Label the vertices of $\mathcal{T}\left(c_{1}\right)$ and $\mathcal{T}\left(c_{2}\right)$ respectively 123 and 145 . As $\sigma_{\mathcal{T}}\left(c_{1}, c_{2}\right)=1, \mathcal{T}\left(c_{1}\right)$ and $\mathcal{T}\left(c_{2}\right)$ cross transversally at the vertex 1 . Let $a=23 \cap 14$. By relabelling the vertices if necessary, one may assume that the arc $2 a$ contains no crossing points. The curves $1 a 3=123+12 a$ and $12 a 45=145+12 a$ are still $\mathbb{Z}_{2}$-homologous and so they must have zero intersection number. However, they touch at the points 1 and $a$, and intersect transversally three times, which is impossible. 
Proposition. Conjecture 2 is true for all graphs $G$ with $n \leq 5$ and $m \leq 9$.

Proof. The cases $n \leq 4$ are given directly by Theorem 2(a). Suppose that $\mathcal{T}: G \rightarrow M_{g}$ is a thrackle with $n=5$. Since $G$ is simple, $G$ is a subgraph of $K_{5}$. Without loss of generality, we may assume that $G$ has no vertices of index 1 . We are required to show that:

(a) if $m \geq 6$, then $G$ cannot be thrackled in the plane,

(b) if $m \geq 8$, then $G$ cannot be thrackled on the torus,

To treat case (a), it suffices to note that if $m \geq 6$, then $G$ either has a 4-cycle or at least two 3-cycles. To deal with case (b), note that on the torus, $H_{1}\left(\mathbb{T}^{2}, \mathbb{Z}_{2}\right)=\mathbb{Z}_{2}^{2}$, and so there are precisely four distinct $\mathbb{Z}_{2}$-homology classes:

$$
\left(\begin{array}{l}
0 \\
0
\end{array}\right),\left(\begin{array}{l}
1 \\
0
\end{array}\right),\left(\begin{array}{l}
0 \\
1
\end{array}\right),\left(\begin{array}{l}
1 \\
1
\end{array}\right) .
$$

So by Lemma 5, a thrackle on the torus can have at most four 3-cycles. Suppose that $m=8$. So $G$ is obtained from $K_{5}$ by deleting two edges. There are only two such graphs, according to whether or not the deleted edges share a common vertex. In the first case,
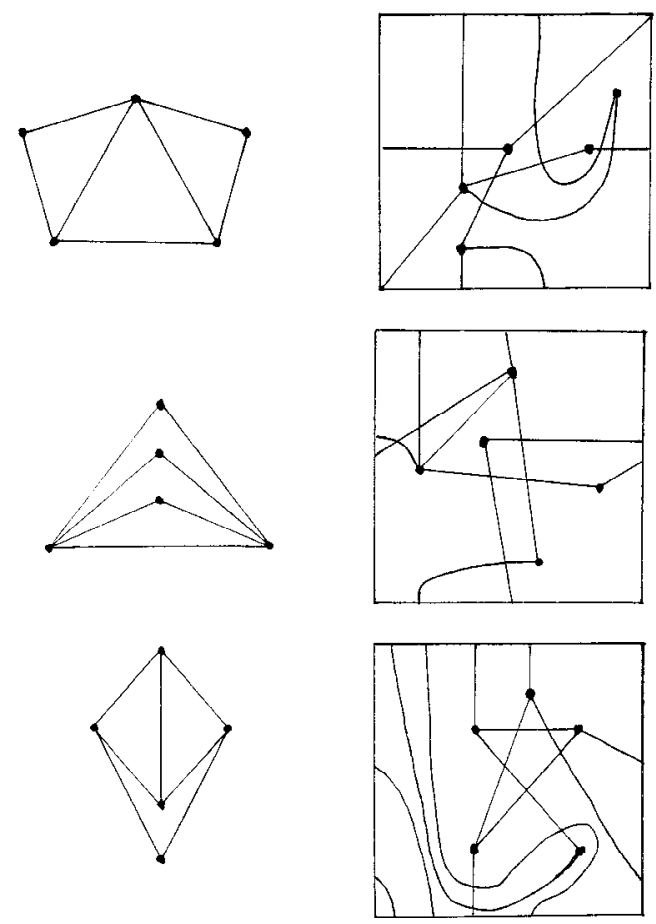

Fig. 14. Thrackles on the torus. 


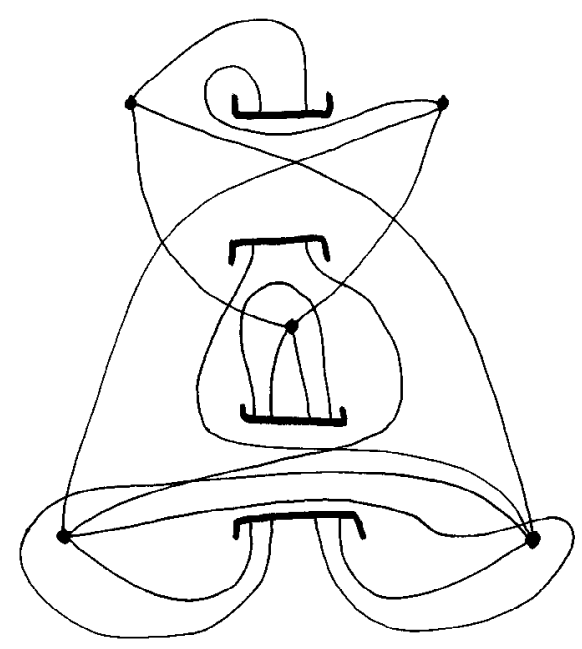

Fig. 15. $K_{5} \backslash\{$ one edge $\}$ thrackled on the 2-torus.

$G$ has five 3-cycles, and so $G$ cannot be thrackled on the torus. In the second case, $G$ has four 3-cycles whose sum is a 4-cycle. Suppose that $G$ can be thrackled on the torus. By Lemma $5, G$ must have precisely one 3 -cycle of each of the four $\mathbb{Z}_{2}$-homology types. So the sum of the 3 -cycles is a 4 -cycle which is zero in $\mathbb{Z}_{2}$-homology. This contradicts Lemma 5(a).

Remark. In support of Conjecture 2, we remark that all graphs with five vertices and seven edges can be thrackled on the torus (see Fig. 14) and the connected graph with five vertices and nine edges, $K_{5} \backslash\{$ one edge\}, can be thrackled on the 2-torus (see Fig. 15). It would be interesting to show that $K_{5}$ cannot be thrackled on the 2-torus.

\section{References}

[CFG] H.T. Croft, K.J. Falconer and R.K. Guy, Unsolved Problems in Geometry, Springer-Verlag, New York, 1991.

[DFN] B.A. Dubrovin, A.T. Fomenko and S.P. Novikov, Modern Geometry, Part III, Springer-Verlag, New York, 1990.

[Fu] W. Fulton. Algebraic Topology, a First Course, Springer-Verlag, New York, 1995.

[GR] J.E. Green and R.D. Ringeisen, Combinatorial drawings and thrackle surfaces, in Graph Theory, Combinatorics, and Algorithms, Vols. 1, 2, Wiley-Interscience, New York, 1995, pp. 999-1009.

[GT] J.L. Gross and T.W. Tucker, Topological Graph Theory, Wiley, New York, 1987.

[LPS] L. Lovász, J. Pach and M. Szegedy, On Conway's thrackle conjecture, Discrete Comput. Geom., 18 (1997), 369-376.

[PA] J. Pach and P.K. Agarwal, Combinatorial Geometry, Wiley, New York, 1995.

[PRS] B.L. Piazza, R.D. Ringeisen and S.K. Stueckle, Subthrackleable graphs and four cycles, Discrete Math. 127 (1994), 265-276. 
[Ri] R.D. Ringeisen, Two old extremal graph drawing conjectures: progress and perspectives, Congr. Numer. 115 (1996), 91-103.

[ST] H. Seifert and W. Threlfall, A Textbook of Topology, Academic Press, London, 1980.

[Wo1] D.R. Woodall, Thrackles and deadlock, in Combinatorial Mathematics and Its Applications (D. Welsh, ed.), Academic Press, New York, 1971, pp. 335-347.

[Wo2] D.R. Woodall, Unsolved problems, in Combinatorics (Proc. Conf. Combinatorial Math., Math. Inst., Oxford), Inst. Math. Appl. Southend-on-Sea, 1972, pp. 359-363.

Received July 23, 1998, and in revised form January 1, 1999. 